\title{
Anti-microRNA-132 causes sevoflurane-induced neuronal apoptosis via the PI3K/AKT/FOXO3a pathway
}

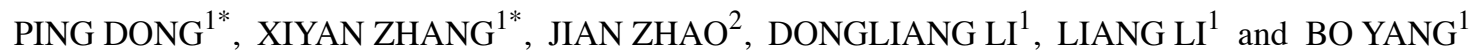 \\ ${ }^{1}$ Department of Anesthesiology, Qilu Hospital, Shandong University, Jinan, Shandong 250012; \\ ${ }^{2}$ Department of Anesthesiology, The People's Hospital of Chiping, \\ Chiping, Shandong 252100, P.R. China
}

Received June 21, 2017; Accepted July 10, 2018

DOI: $10.3892 /$ ijmm.2018.3895

\begin{abstract}
In the present study, the mechanisms underlying the protective effects of microRNA-132 (miRNA-132) on sevoflurane-induced neuronal apoptosis were investigated. Reverse transcription-quantitative polymerase chain reaction and gene microarray hybridization were used to analyze alterations in microRNA levels. Cell viability, apoptosis and caspase-3/9 activity were measured using MTT, flow cytometry and caspase-3/9 activity kits. Immunofluorescence staining and western blot analysis were used to measure protein expression of phosphoinositide 3-kinase (PI3K) and phosphorylated (p-)AKT, forkhead box O3a (FOXO3a). In sevoflurane-induced rats, the expression of miRNA-132 was downregulated, compared with that in negative control rats. The downregulation of miRNA-132 increased neuronal apoptosis and the upregulation of miRNA-132 inhibited neuronal apoptosis in the sevoflurane-induced in vitro model. The downregulation of miRNA-132 suppressed the protein expression of PI3K and p-AKT, and suppressed the protein expression of FOXO3a in the sevoflurane-induced in vitro model. The PI3K inhibitor increased the effects of anti-miRNA-132 on neuronal apoptosis through the AKT/FOXO3a pathway in the sevoflurane-induced in vitro model. The promotion of FOXO3a inhibited the effects of anti-miRNA-132 on neuronal apoptosis through the AKT/FOXO3a pathway in the sevoflurane-induced in vitro model. These data suggested that miRNA-132 caused sevoflurane-induced neuronal apoptosis via suppression of the PI3K/AKT/FOXO3a pathway.
\end{abstract}

Correspondence to: Ms. Bo Yang, Department of Anesthesiology, Qilu Hospital, Shandong University, 107 Wenhua West Road, Jinan, Shandong 250012, P.R. China

E-mail: bpy94861763@126.com

*Contributed equally

Key words: microRNA-132, sevoflurane, neuronal apoptosis, phosphoinositide 3-kinase, AKT, forkhead box O3a

\section{Introduction}

Surgery has developed rapidly in recent years, which has led to a gradual increase in the proportion of patients receiving general anesthesia (1). General anesthesia has been applied for almost two centuries. However, the precise mechanism of action remains to be fully elucidated (2). Sevoflurane has the advantages of rapid induction and resuscitation. In addition, it has a small influence on liver and renal function, and hemodynamics (2). Therefore, it is applied extensively clinically. It is considered that intravenous and inhaled drugs can induce the apoptosis of central nervous system cells (2). In particular, anesthetic drugs affect the construction of the central nervous network at a critical stage of brain tissue development (2). In addition, they can impair learning and memory function in the future (1). Preclinical experiments have indicated that the neurotoxicity of sevoflurane in general anesthesia on the developing brain is associated with neuronal apoptosis and subsequent cognitive impairment (3). Consequently, treatment against neuronal apoptosis may alleviate sevoflurane-induced neurocognitive impairment (4).

Cell apoptosis, the most commonly observed sevoflurane-induced injury, is associated with complicated and diverse mechanisms (5). The phosphoinositide 3-kinase (PI3K)/Akt signal transduction pathway is the most important among numerous signal transduction pathways in the cell apoptotic mechanism (5). Its mechanism of inhibiting cell apoptosis may be realized through regulating apoptin and apoptotic genes (6). Alternatively, it can be achieved through affecting the corresponding changes of mitochondrion and endoplasmic reticulum. Neurotrophin can activate the PI3K/Akt signal transduction pathway (7). The nuclear transcription factor forkhead box O3a (FOXO3a) is a downstream substrate of the PI3K/Akt signaling pathway (8). It is closely associated with cell proliferation, aging, apoptosis, differentiation and tumor genesis (8).

MicroRNAs (miRNAs) are highly conserved, non-coding, single-stranded RNAs, which are only 21-24 nucleotides in length (9). They can inhibit target gene expression at the transcriptional level (10). In addition, they are involved in regulating almost all physiopathological processes, including growth, development, inflammation, swelling and neuro- 
degenerative disease (10). The expression of miRNAs is time- and space-specific. The expression level of a specific miRNA may be different under various developmental stages. Alternatively, it may be different under different histological or pathological conditions (9). Wang et al showed that miR-132 affects glioma cell growth (11). Leinders et al also reported that the expression of miR-132-3p is associated with chronic neuropathic pain (12). Yao et al suggested that the hypoxia-induced upregulation of miR-132 promoted Schwann cell migration and facilitated peripheral nerve regeneration (13). Therefore, miR-32 has become a focus of investigations in the medical community. In the present study, the mechanisms underlying the protective effects of miRNA-132 on sevoflurane-induced neuronal apoptosis were investigated.

\section{Materials and methods}

Animals and anesthesia treatment. The study protocol was approved by the Animal Use and Care Committee for Research and Education of Shandong University (Shandong, China). Sprague-Dawley rats (male, 220-250 g, 8-10 weeks) were purchased from Shandong Animal Laboratory (Shandong, China) and housed at $22 \pm 1^{\circ} \mathrm{C}$ under a $12-\mathrm{h}$ light/dark cycle (7:00 am-7:00 pm). A total of 12 rats were randomly assigned into two groups: Normal control group $(n=6)$ and sevoflurane-induced model group $(n=6)$. In the sevoflurane-induced model group, the rats were placed into a chamber and exposed to $2 \%$ sevoflurane for $6 \mathrm{~h}$. After $24 \mathrm{~h}$, the hippocampus of rats in all groups was analyzed following sacrifice of the rats.

Hematoxylin and eosin staining. The histology of the hippocampus and the apoptotic status were examined using hematoxylin staining. The brains were fixed in $4 \%$ paraformaldehyde for $24 \mathrm{~h}$, paraffin-embedded and cut into 5 - $\mu \mathrm{m}$-thick sections. The tissue samples were then stained with hematoxylin and eosin, and the sections were examined under an optical microscope using fluorescence (BX53; Olympus, Tokyo, Japan).

Cell culture, transfection and treatment. The human $\mathrm{H} 4$ neuroglioma cell line was obtained from the Cell Bank of the Chinese Academy of Sciences (Shanghai, China) and cultured in RPMI-1640 (HyClone; GE Healthcare Life Sciences, Logan, UT, USA) supplemented with $10 \%$ fetal bovine serum (Gibco; Thermo Fisher Scientific, Inc., Waltham, MA, USA) in a humidified atmosphere of $5 \% \mathrm{CO}_{2}$ at $37^{\circ} \mathrm{C}$. The FOXO3 plasmid, miR-132 mimic, anti-miR-132 mimic and control mimic were synthesized by GenePharma Co., Ltd. (Shanghai, China). The cells were plated at $70-80 \%$ confluence in a 6 -well plate and were transfected with 100 ng mimics using Lipofectamine 2000 (Invitrogen; Thermo Fisher Scientific, Inc.) according to the manufacturer's protocol.

Reverse transcription-quantitative polymerase chain reaction (RT-qPCR) analysis and gene microarray hybridization. Total RNA was extracted from the tissue and cells using an RNA simple total RNA kit (Tiangen Biotech Co., Ltd., Beijing,
China), and $200 \mathrm{nM}$ total RNA was reverse transcribed into cDNA using Super M-MLV reverse transcriptase (BioTeke Corporation, Beijing, China) at $37^{\circ} \mathrm{C}$ for $1 \mathrm{~h}$ and $84^{\circ} \mathrm{C}$ for 5 min. RT-qPCR analysis was performed in an ABI Prism 7500 Real-time PCR system using SYBR-Green master mix (Solarbio Science and Technology Co., Ltd., Beijing, China) with the following cycling parameters: $10 \mathrm{~min}$ at $95^{\circ} \mathrm{C}$, followed by 40 cycles of $30 \mathrm{sec}$ at $95^{\circ} \mathrm{C}$ and $30 \mathrm{sec}$ at $60^{\circ} \mathrm{C}$. The following primers were used in the present study: miR-132: 5'-CCGCGTCTCCAGGGCAAC-3' and 5'-CCTCCGGTT CCCACAGTAACAA-3'; and U6: 5'-TGGTATTCGTGGAAG GACTCATGAC-3' and 5'-ATGCCAGTGAGCTTCCCGTTC AGC-3'. Analysis of relative gene expression data was quantified using the $2^{-\Delta \Delta \mathrm{Ct}}$ method (14).

Total RNA was labeled using Cyanine-5-CTP and hybridized to the SurePrint G3 Mouse Whole Genome GE Microarray G4852A platform with an equimolar concentration of Cyanine-3-CTP-labelled universal rat reference (Stratagene; Agilent Technologies, Inc., Santa Clara, CA, USA). Images were quantified and feature-extracted using Agilent Feature Extraction software (version A.10.7.3.1; Agilent Technologies, Inc.).

Cell viability assays. Cell viability $\left(1 \times 10^{3}\right.$ cell/well) was measured using an MTT assay $(20 \mu 1 ; 5 \mathrm{mg} / \mathrm{ml})$ at 96 -well plate and incubated for $4 \mathrm{~h}$ at $37^{\circ} \mathrm{C}$. After $4 \mathrm{~h}$, the medium was removed, and $150 \mu \mathrm{l}$ DMSO was added to the cells and incubated for $20 \mathrm{~min}$ for $37^{\circ} \mathrm{C}$. The absorbance was measured using the ELX-800 absorbance microplate reader (Bio-Tek Instruments, Inc., Winooski, VT, USA) at $450 \mathrm{~nm}$.

Cell viability was measured according to lactate dehydrogenase (LDH) activity (Beyotime Institute of Biotechnology, Haimen, China) and the absorbance was measured using the ELX-800 absorbance microplate reader (Bio-Tek Instruments, Inc.) at $450 \mathrm{~nm}$.

Analysis of apoptosis by flow cytometry and caspase-3/9 activity. The cells $\left(1 \times 10^{6}\right.$ cell/well) were washed with PBS and resuspended in $500 \mu \mathrm{l}$ binding buffer (BD Biosciences, Franklin Lakes, NJ, USA). The cells were stained with anti-Annexin V-FITC antibody (5 $\mu \mathrm{l})$ and PI (5 $\mu \mathrm{l})$ for $15 \mathrm{~min}$ at room temperature in the dark. The apoptotic rates were measured using flow cytometric analysis on a FACSCalibur flow cytometer (BD Biosciences).

The cells were used to extract total proteins using RIPA lysis buffer (Beyotime Institute of Biotechnology) and protein concentrations were determined using BCA protein assays (Beyotime Institute of Biotechnology). The proteins $(10 \mu \mathrm{g})$ were used to measure the activity of caspase-3/9 using caspase- 3 or caspase- 9 activity kits (Beyotime Institute of Biotechnology). The absorbance was measured using the ELX-800 absorbance microplate reader (Bio-Tek Instruments, Inc.) at $405 \mathrm{~nm}$.

Immunofluorescence staining. The cells were fixed using 4\% paraformaldehyde for $15 \mathrm{~min}$ at room temperature and permeabilized with $0.1 \%$ Triton X-100 (Beyotime Institute of Biotechnology) for $15 \mathrm{~min}$ at room temperature. The cells $\left(1 \times 10^{4}\right.$ cell/well $)$ were then incubated with 1:100 diluted anti-nuclear factor $(\mathrm{NF})-\kappa \mathrm{B}$ p 65 antibody 


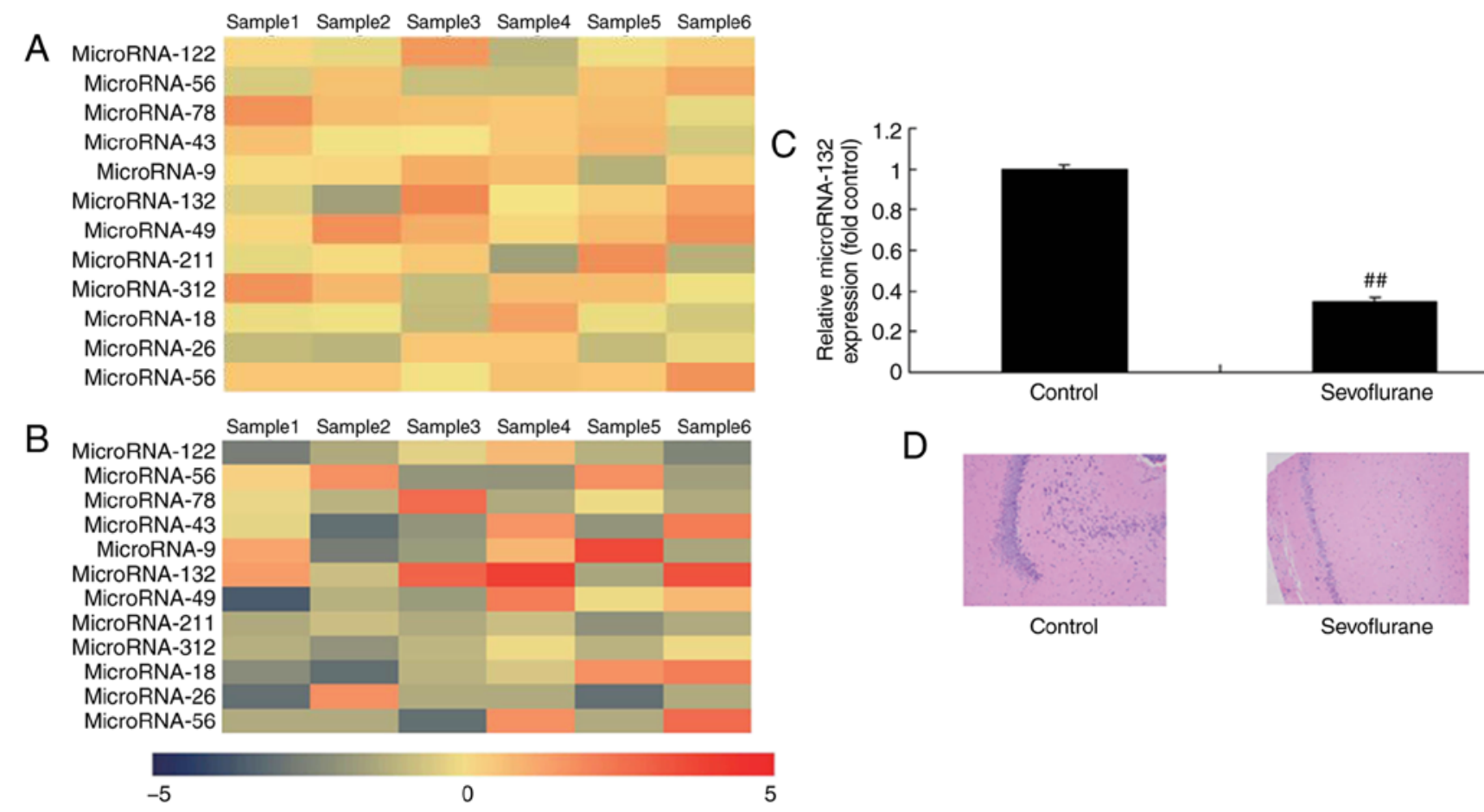

Figure 1. Expression of miRNA-132 in sevoflurane-induced rats. Expression of miRNAs in the (A) control group and (B) sevoflurane-induced rat group were evaluated using a gene chip assay. Each rat was labeled as sample 1-6. Expression of miRNA-132 was determined using (C) reverse transcription-quantitative polymerase chain reaction analysis and (D) in the hippocampus using a hematoxylin and eosin staining assay (magnification, x100). Values are expressed as the mean \pm standard deviation $(\mathrm{n}=6) .{ }^{\# \#} \mathrm{P}<0.01$, compared with the control group. Control, normal rat; sevoflurane, sevoflurane-induced rat. miRNA, microRNA.

(1:100; cat. no. sc-8008; Santa Cruz Biotechnology, Inc., Dallas, TX, USA), overnight at $4^{\circ} \mathrm{C}$ and incubated with FITC-labeled goat anti-rabbit secondary antibody $(1: 200$; cat. no. A0562; Beyotime Institute of Biotechnology) for $1 \mathrm{~h}$ at room temperature. The cells were then stained with DAPI for $30 \mathrm{~min}$ at room temperature in the dark. The cells were observed under a fluorescence microscope (BX53; Olympus).

Western blot analysis. The cells were used to extract total proteins using RIPA lysis buffer (Beyotime Institute of Biotechnology) and protein concentration was determined using BCA protein assays (Beyotime Institute of Biotechnology). The proteins $(40 \mu \mathrm{g})$ from each sample were separated by $10 \%$ SDS-PAGE and transferred onto a PVDF membrane (EMD Millipore, Bedford, MA, USA). The membrane was blocked with $5 \%$ non-fat milk for $1 \mathrm{~h}$ at $37^{\circ} \mathrm{C}$ and incubated with the following specific primary antibodies: B-cell lymphoma 2 (Bcl-2)-associated X protein (Bax, cat. no. sc-6236; 1:500; Santa Cruz Biotechnology, Inc.), PI3K (cat. no. sc-7174; 1:500; Santa Cruz Biotechnology, Inc.), phosphorylated (p-)AKT (sc-7985-R; 1:300; Santa Cruz Biotechnology, Inc.), FOXO3a (cat. no. 12829; 1:2,000; Cell Signaling Technology, Inc., Danvers, MA, USA) and GAPDH (cat. no. sc-25778; 1:2,000; Santa Cruz Biotechnology, Inc.) at $4^{\circ} \mathrm{C}$ overnight. Subsequently, the membrane was incubated with corresponding horseradish peroxidase-conjugated secondary antibodies (cat. no. sc-2004; 1:5,000; Santa Cruz Biotechnology, Inc.) at $37^{\circ} \mathrm{C}$ for $1 \mathrm{~h}$. The blots of the proteins were visualized using Enhanced Chemiluminescence reagents (Beyotime Institute of Biotechnology) and quantified using
Image Lab 3.0 (Bio-Rad Laboratories, Inc., Hercules, CA, USA).

Statistical analysis. Values are expressed as the mean \pm standard deviation using SPSS software (version 17.0; SPSS, Inc., Chicago, IL, USA). All data were analyzed using Student's t-test or one-way analysis of variance with Bonferroni's post hoc test. $\mathrm{P}<0.05$ was considered to indicate a statistically significant difference.

\section{Results}

Expression of miR-132 in sevoflurane-induced rats. Gene chip and RT-qPCR analyses were used to measure the expression of miRNA-132 in sevoflurane-induced rats, and it was found that the expression of miRNA-132 was downregulated in sevoflurane-induced rats, compared with that in the normal group (Fig. 1A and B). A high level of neuronal apoptosis was observed in the sevoflurane-induced rats, compared with the normal group (Fig. 1C). These data suggested that the downregulation of miR-132 may be associated with sevoflurane-induced neuronal apoptosis.

miR-132 affects neuronal cell growth in a sevoflurane-induced in vitro model. The expression levels of miR-132 in the $\mathrm{H} 4$ cells transfected with miR-132, anti-miR-132 mimic or mimic control were determined by RT-qPCR analysis. As shown in Fig. 2A and B, miR-132 and anti-miR-132 mimic increased and inhibited the expression level of miR-132 in the sevoflurane-induced cells, respectively, compared with the mimic control group. The overexpression of miR-132 and 

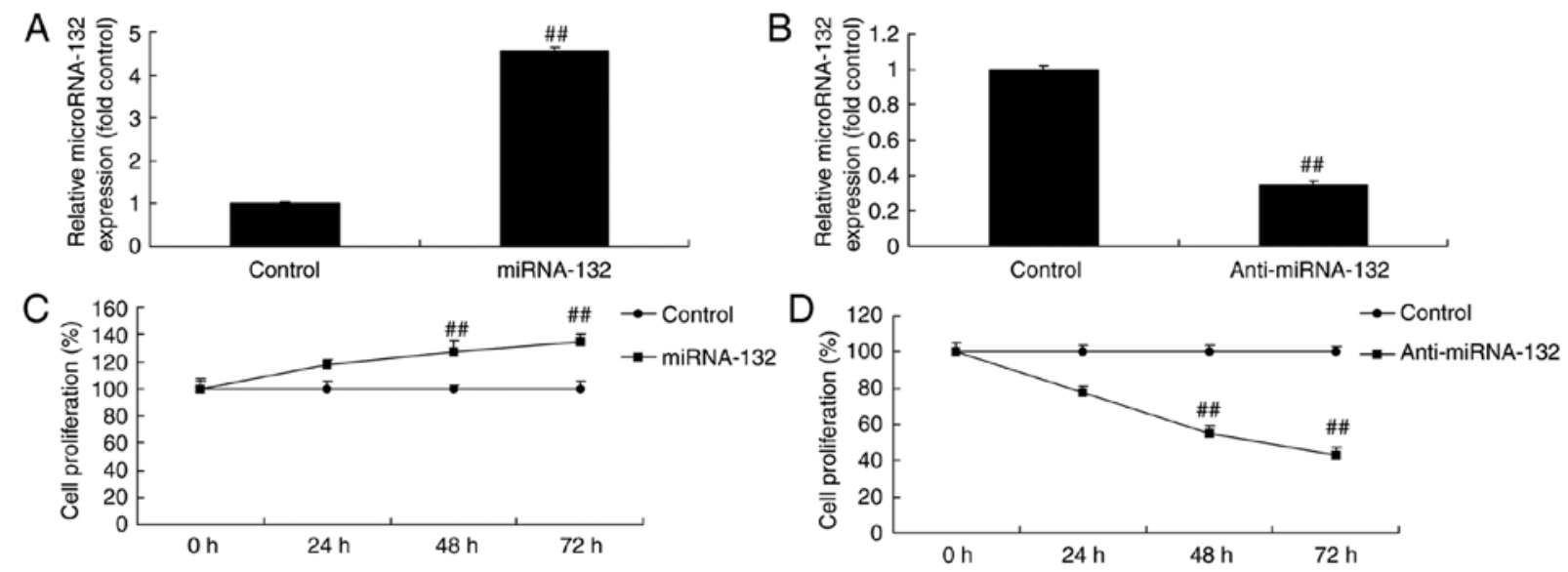

Figure 2. miRNA-132 affects neuronal cell growth in a sevoflurane-induced in vitro model. Expression of miRNA-132 determined using reverse transcription-quantitative polymerase chain reaction following transfection with (A) miRNA-132 and (B) anti-miRNA-132 mimics. Cell proliferation in cells transfected with (C) miRNA-132 and (D) anti-miRNA-132 mimics. Values are expressed as the mean \pm standard deviation $(\mathrm{n}=3)$. ${ }^{\# \prime} \mathrm{P}<0.01$, compared with the control group. miRNA, microRNA; control, negative control; miRNA-132, overexpression of miRNA-132; anti-miRNA-132, downregulated expression of miR-132.
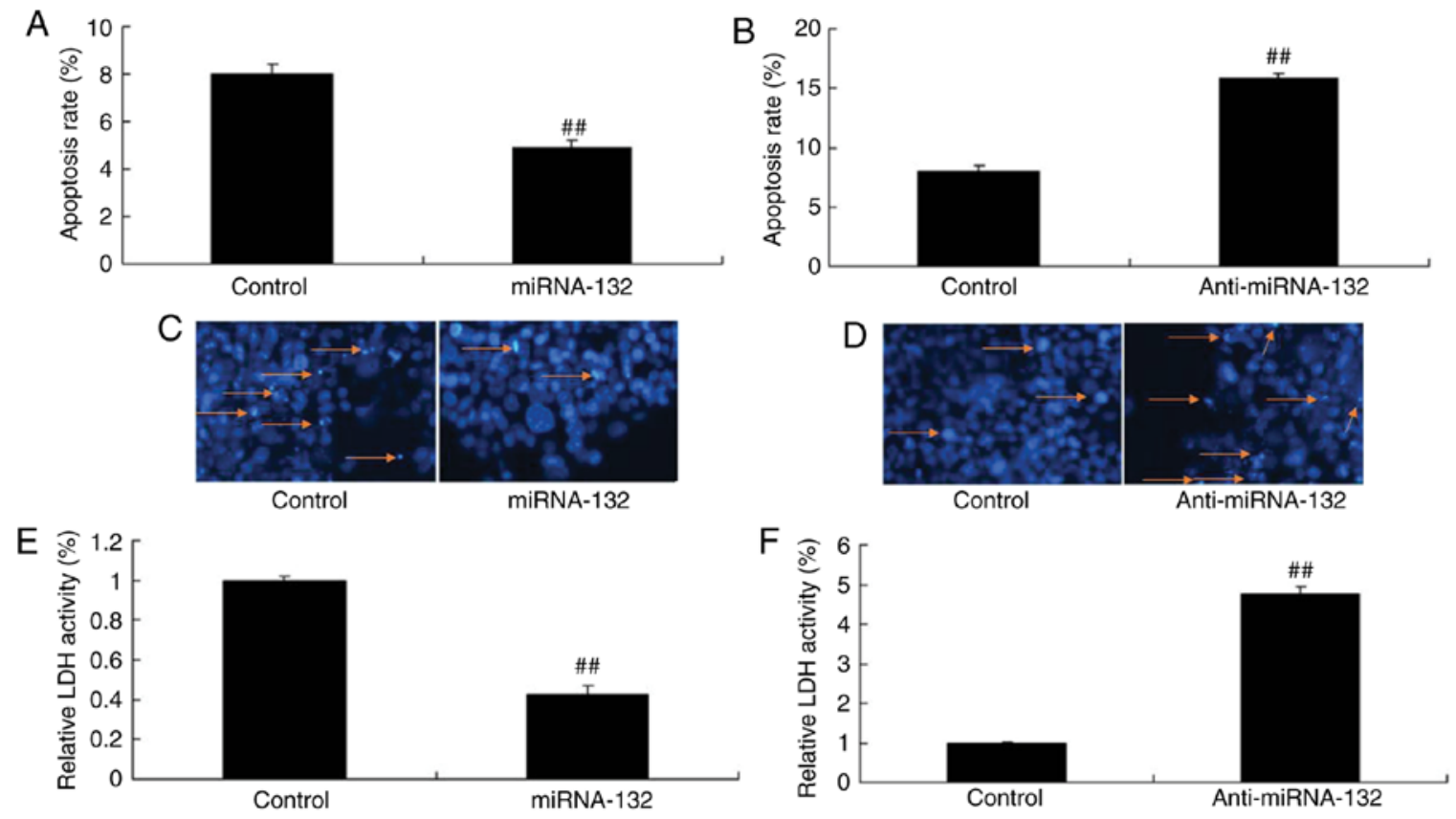

Figure 3. miRNA-132 affects neuronal cell apoptosis in a sevoflurane-induced in vitro model. Cell apoptosis in was determined using flow cytometry in (A) miRNA-132 and (B) anti-miRNA-132 groups, and by staining using DAPI (magnification, x10) in (C) miRNA-132 and (D) anti-miRNA-132 groups Activity of LDH in (E) miRNA-132 and (F) anti-miRNA-132 group. Values are expressed as the mean \pm standard deviation $\left(\mathrm{n}=3\right.$ ). ${ }^{\# \#} \mathrm{P}<0.01$, compared with the control group. miRNA, microRNA; control, control negative group; miRNA-132, overexpression of miRNA-132; anti-miRNA-132, downregulated expression of miRNA-132; LDH, lactate dehydrogenase. Arrows indicate apoptotic nuclei.

downregulation of miR-132 promoted neuronal cell growth and inhibited neuronal cell growth in the sevoflurane-induced in vitro model, respectively, compared with the mimic control group (Fig. 2C and D).

miRNA-132 affects neuronal cell apoptosis in a sevoflurane-induced in vitro model. The overexpression of miRNA-132 and downregulation of miRNA-132 reduced and increased the apoptotic rate in the sevoflurane-induced in vitro model, respectively, compared with the mimic control group (Fig. 3A and B). DAPI was used to stain cells, and it was also found that the overexpression of miRNA-132 and downregulation of miRNA-132 inhibited and increased cell apoptosis in the sevoflurane-induced in vitro model, respectively, compared with the mimic control group (Fig. 3C and D). The activity of LDH in the cells transfected with miR-132 was reduced and that transfected with anti-miR-132 mimic was increased, compared with that in the mimic control group (Fig. 3E and F).

miRNA-132 affects the expression of Bax and caspase-3/9 in the sevoflurane-induced in vitro model. The overexpression of miRNA-132 suppressed the expression levels of Bax and caspase-3/9, and the downregulation of miRNA-132 induced the expression of Bax and caspase-3/9 in the sevoflurane-induced in vitro model, respectively, compared with that in the mimic control group (Fig. 4A-F). 
A
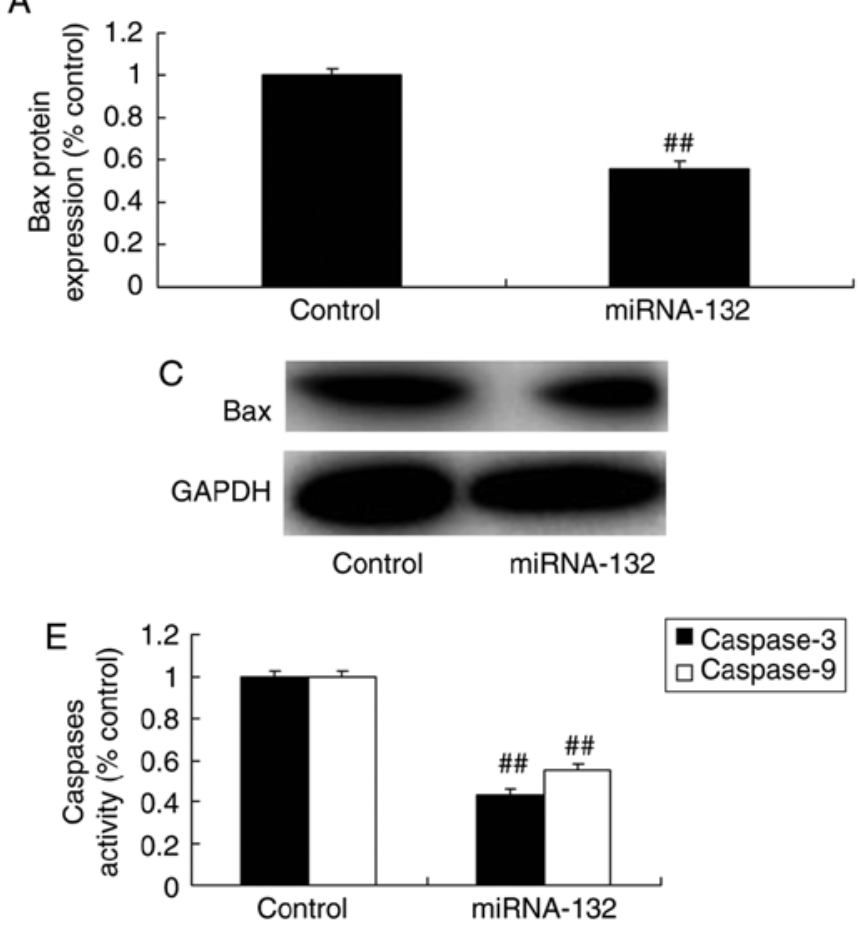
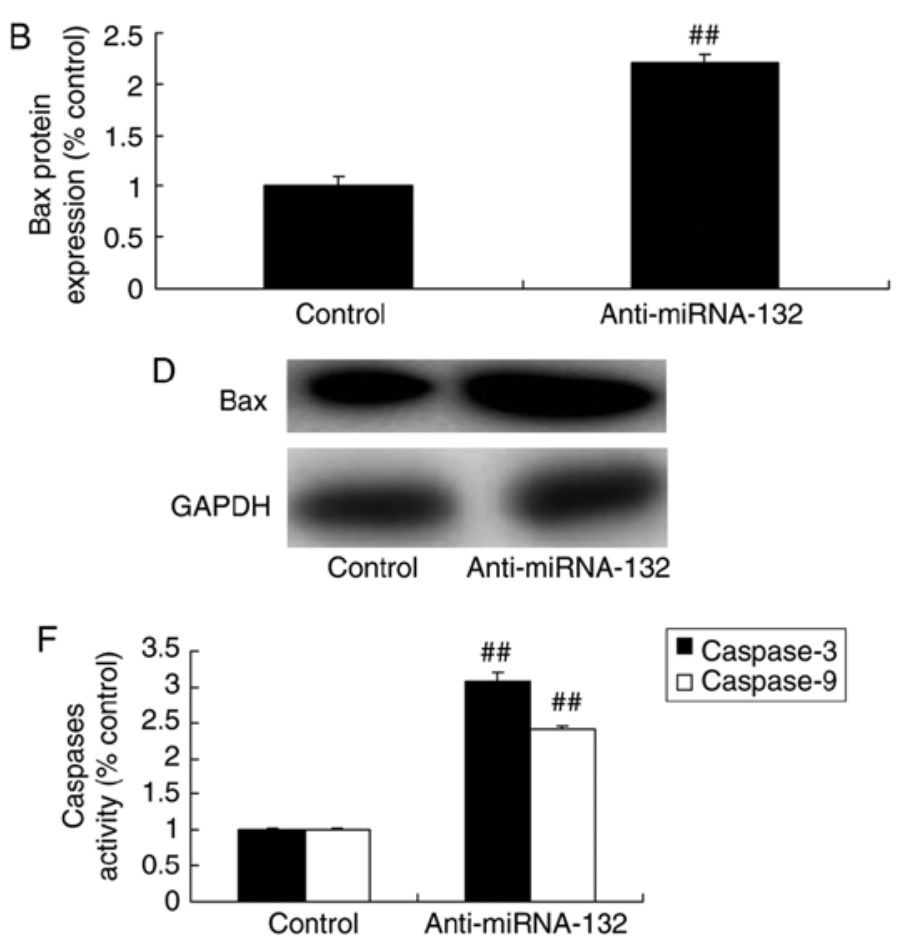

Figure 4. miRNA-132 affects expression levels of Bax and caspase-3/9 in a sevoflurane-induced in vitro model. Protein expression of Bax in (A) miRNA-132 and (B) anti-miRNA-132 groups was determined using statistical analysis. Western blot assays for the (C) miRNA-132 and (D) anti-miRNA-132 groups.

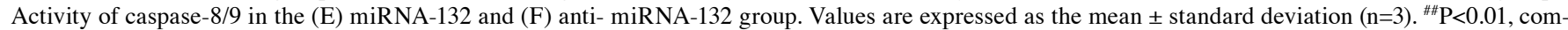
pared with the control group. miRNA, microRNA; control, negative control; miRNA-132, overexpression of miRNA-132; anti-miRNA-132, downregulated expression of miRNA-132; Bax, B-cell lymphoma 2-assocated X protein.

miRNA-132 affects the PI3K/AKT/FOXO3a pathway in the sevoflurane-induced in vitro model. To investigate whether miRNA-132 exerts its cytoprotective effect via the PI3K/AKT/FOXO3a pathway, the protein expression levels of PI3K, p-AKT and FOXO3a were measured in the sevoflurane-induced in vitro model. The overexpression of miRNA-132 induced the PI3K/AKT/FOXO3a pathway in the sevoflurane-induced in vitro model (Fig. 5A-D). The downregulation of miRNA-132 inhibited the PI3K/AKT/FOXO3a pathway in the sevoflurane-induced in vitro model (Fig. 5E-H), as shown in the immunofluorescence staining (Fig. 5I and J).

PI3K inhibitor increases the effects of anti-miRNA-132 on the AKT/FOXO3a pathway in a sevoflurane-induced in vitro model. Thepresent study alsoexamined whetherPI3K inhibitorincreased the effects of anti-miRNA-132 on the PI3K/AKT/FOXO3a pathway by using the PI3K inhibitor, LY294002 (100 nM), to reduce the PI3K/AKT/FOXO3a pathway in the sevoflurane-induced in vitro model following anti-miRNA-132. As shown in Fig. 6A-D, the PI3K inhibitor suppressed the PI3K/AKT/FOXO3a pathway in the sevoflurane-induced in vitro model following anti-miRNA-132 treatment, compared with the anti-miRNA-132 only-treated group.

PI3K inhibitor increases the effects of anti-miRNA-132 on neuronal apoptosis in the sevoflurane-induced in vitro model. The PI3K inhibitor increased the effects of anti- miRNA-132 on the inhibition of neuronal cell growth, activation of cell apoptosis, activity of LDH, and induction of the expression of Bax and caspase-3/9 in the sevoflurane-induced in vitro model, compared with the effects in the anti-miRNA-132 group (Figs. 7A-D and 8A-C).

Promotion of FOXO3a inhibits the effects of anti-miRNA-132 on neuronal apoptosis through the FOXO3a pathway in a sevoflurane-induced in vitro model. To confirm the activation of the FOXO3a signaling pathway following anti-miRNA-132 treatment in in sevoflurane-induced in vitro model, western blot analysis was performed. The FOXO3 plasmid induced the protein expression of FOXO3a and suppressed the expression of Bax and caspase-3/9 in the sevoflurane-induced in vitro model induced by anti-miRNA-132, compared with the expression levels in the anti-miRNA-132 group (Fig. 9A-D).

Promotion of FOXO3a inhibits the effects of anti-miRNA-132 on neuronal apoptosis through the AKT/FOXO3 a pathway in a sevoflurane-induced in vitro model. Finally, the promotion of FOXO3a inhibited the effects of anti-miRNA-132 on the inhibition of neuronal cell growth, activation of cell apoptosis and activity of caspase-3/9 in the sevoflurane-induced in vitro model, compared with the effects in the anti-miRNA-132 group (Fig. 10A-D).

\section{Discussion}

Sevoflurane is a common inhaled anesthetic gas (15). At present, the problem of whether sevoflurane anesthesia produces neuroprotective or neurotoxic effects is controversial (15). Numerous existing preclinical studies indicate that general inhaled anesthetics, including sevoflurane, induce extensive neuronal 

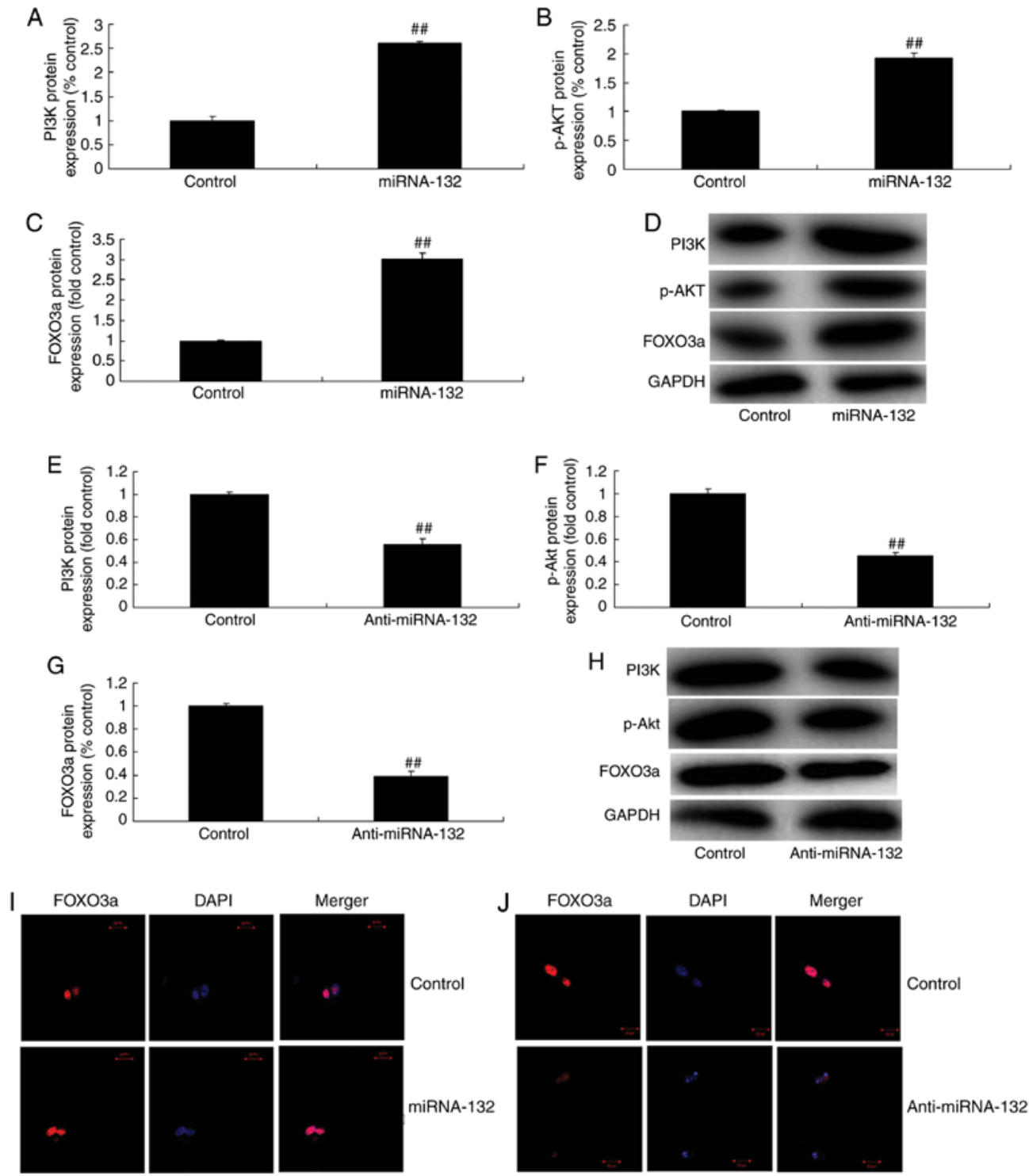

Figure 5. miRNA-132 affects the PI3K/AKT/FOXO3a pathway in a sevoflurane-induced in vitro model. Protein expression levels of (A) PI3K, (B) p-AKT and (C) FOXO3a were determined using statistical analysis. Western blot analysis was used to the determine effects in the (D) overexpression of miRNA-132 group. Protein expression levels of (E) PI3K, (F) p-Akt and (G) FOXO3a were determined using statistical analysis. (H) Western blot analysis following the downregulated expression of miRNA-132. Immunofluorescence staining (magnification, $\mathrm{x} 40$ ) of protein expression following (I and J) the downregulated expression of miRNA-132. Values are expressed as the mean \pm standard deviation $(\mathrm{n}=3) .{ }^{\# \#} \mathrm{P}<0.01$, compared with control group. miRNA, microRNA; control, negative control; miRNA-132, overexpression of miRNA-132; anti-miRNA-132, downregulated expression of miRNA-132.
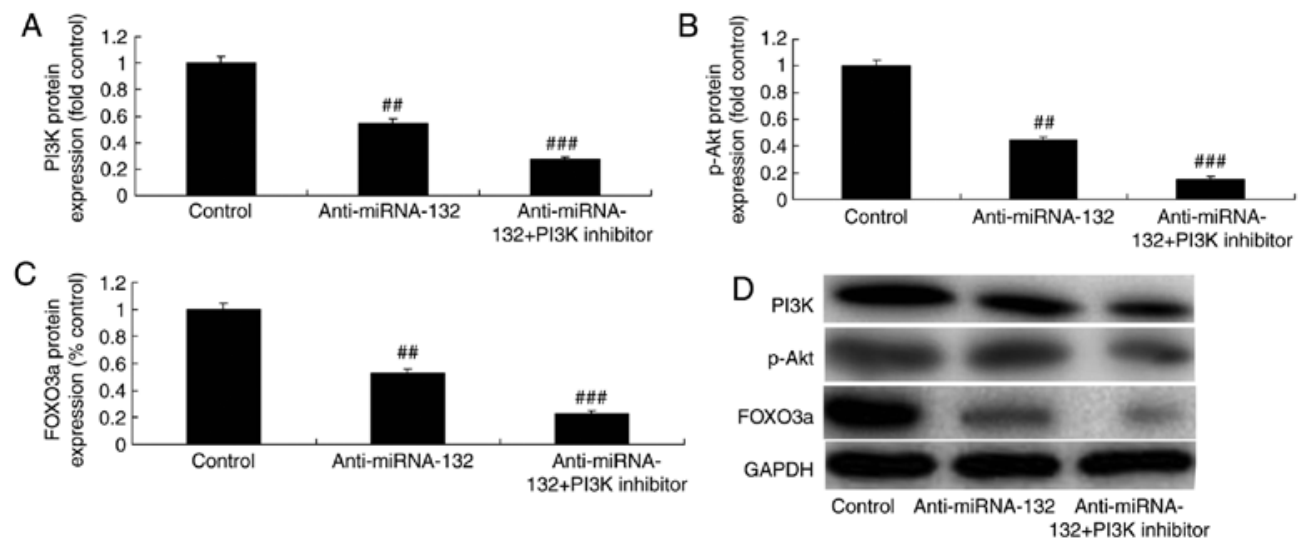

Figure 6. PI3K inhibitor increases the effects of anti-miRNA-132 through the AKT/FOXO3a pathway in a sevoflurane-induced in vitro model. Protein expression of (A) PI3K, (B) p-Akt and (C) FOXO3a, determined via statistical analysis of (D) western blot assays. Values are expressed as the mean \pm standard deviation (n=3). ${ }^{\# \#} \mathrm{P}<0.01$, compared with the control group; ${ }^{\# \# "} \mathrm{P}<0.01$, compared with the anti-miRNA-132 group. miRNA, microRNA; control, negative control; anti-miRNA-132, downregulated expression of miRNA-132; PI3K inhibitor, LY294002; PI3K, phosphoinositide 3-kinase; p-Akt, phosphorylated Akt; FOXO3a, forkhead box O3A. 

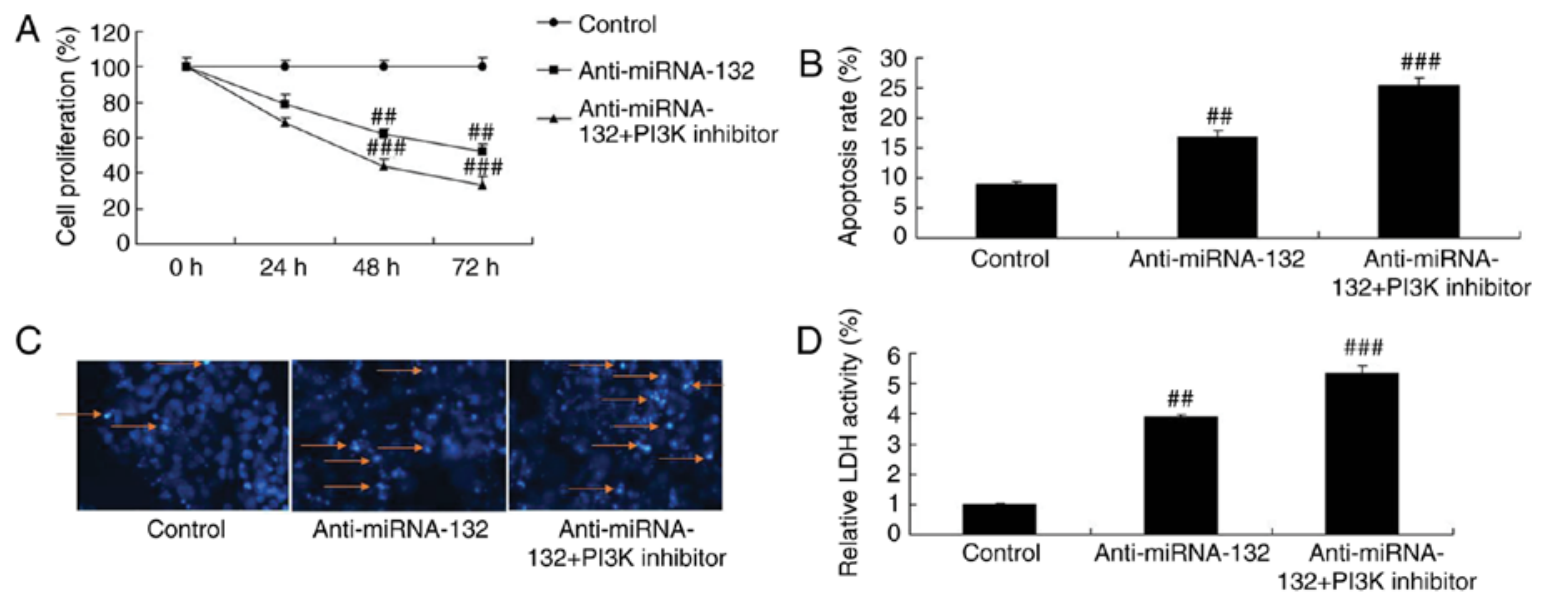

Figure 7. PI3K inhibitor increases the effects of anti-miRNA-132 on neuronal apoptosis in a sevoflurane-induced in vitro model. (A) Cell proliferation and (B) apoptosis, determined using flow cytometry and (C) DAPI staining (magnification, x10). (D) LDH activity. Values are expressed as the mean \pm standard deviation $(\mathrm{n}=3) .{ }^{\# /} \mathrm{P}<0.01$, compared with the control group; ${ }^{\# \# \#} \mathrm{P}<0.01$, compared with the anti-miRNA-132 group. miRNA, microRNA; control, negative control; anti-miRNA-132, downreguated expression of miRNA-132; PI3K inhibitor, LY294002; LDH, lactate dehydrogenase. Arrows indicate apoptotic nuclei.
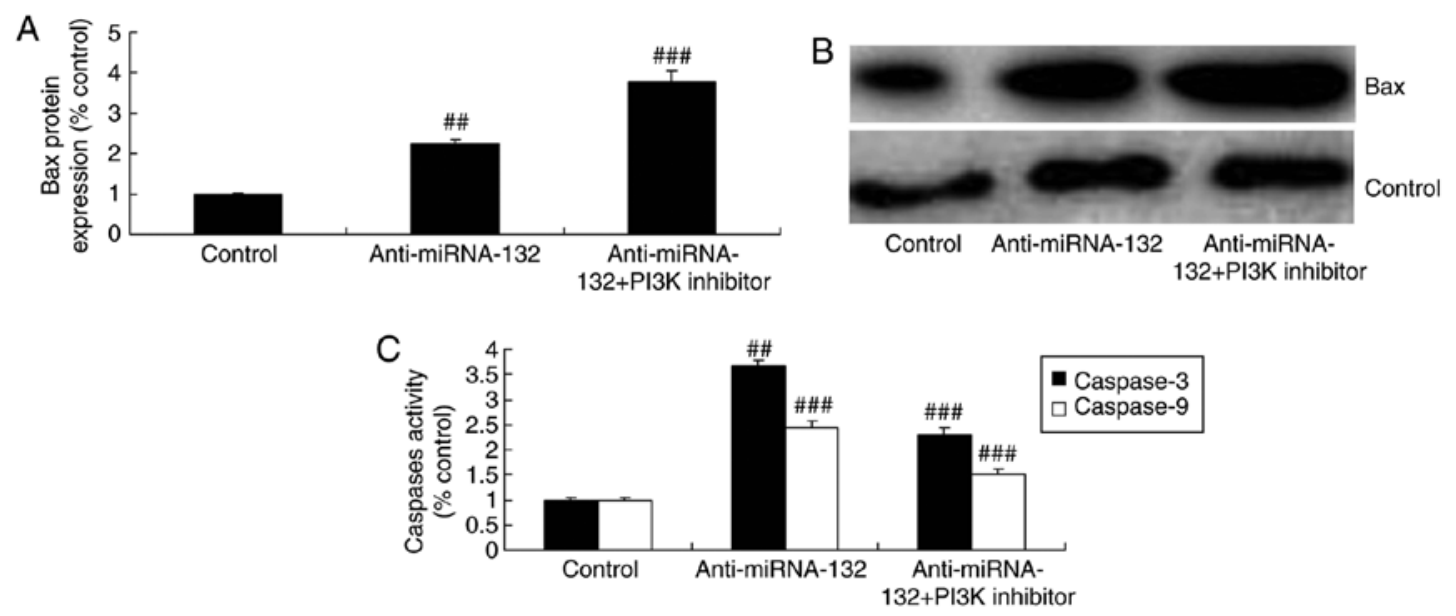

Figure 8. PI3K inhibitor increases the effects of anti-miRNA-132 on the expression of Bax and caspase-3/9 in a sevoflurane-induced in vitro model. Protein expression of Bax, determined via (A) statistical analysis of (B) western blot assays; (C) caspase-8/9 activity. Values are expressed as the mean \pm standard deviation $(\mathrm{n}=3)$. ${ }^{\# \#} \mathrm{P}<0.01$, compared with the control group; ${ }^{\# \# \#} \mathrm{P}<0.01$, compared with the anti-miRNA-132 group. miRNA, microRNA; control, negative control; anti-miRNA-132, downregulated expression of miRNA-132; PI3K inhibitor, LY294002.
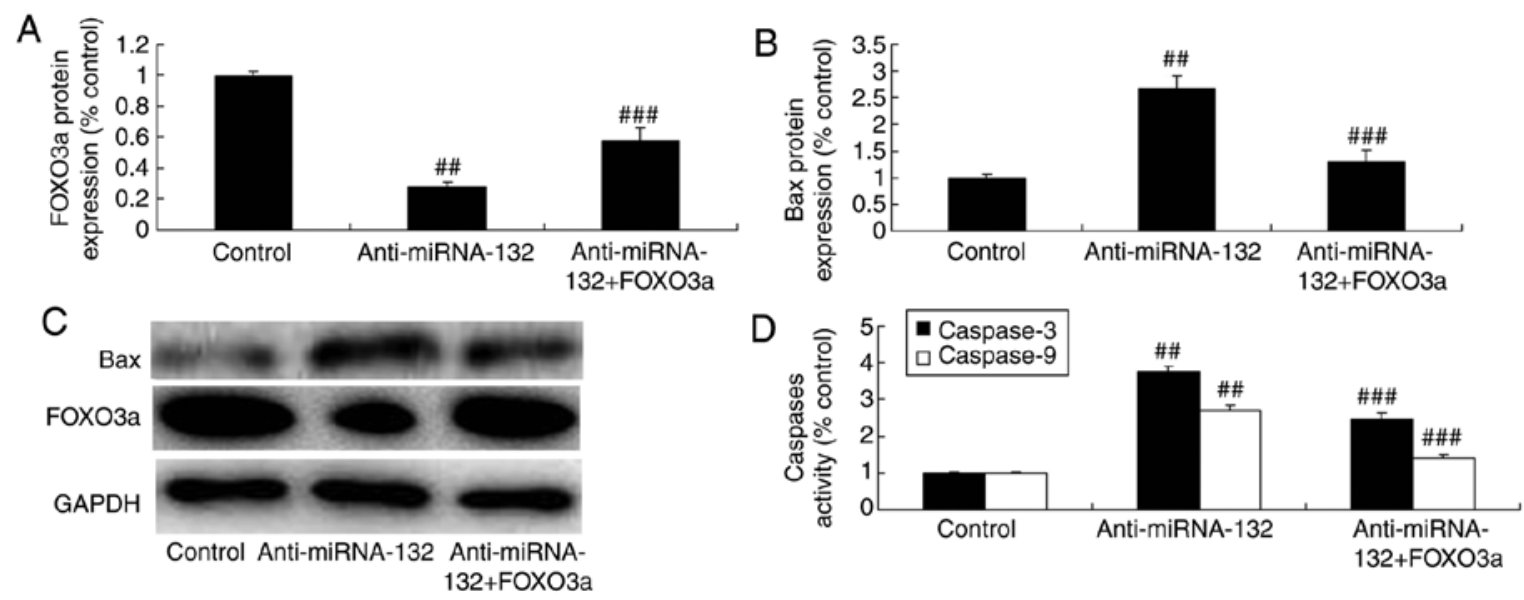

Figure 9. Promotion of FOXO3a inhibits the effects of anti-miRNA-132 on neuronal apoptosis through the FOXO3a pathway in a sevoflurane-induced in vitro model. Statistical analysis of protein expression of (A) FOXO3a and (B) Bax from (C) western blot assays. (D) caspase-8/9 activity. Values are expressed as the mean \pm standard deviation $(\mathrm{n}=3) .{ }^{\# \#} \mathrm{P}<0.01$, compared with the control group; ${ }^{\# \# \#} \mathrm{P}<0.01$, compared with the anti-miRNA-132 group. FOXO3a, forkhead box O31; Bax, B-cell lymphoma 2-associated X protein; miRNA, microRNA; control, negative control; anti-miRNA-132, downregulated expression of miRNA-132; Anti- miRNA-132+FOXO3a, anti-miRNA-132+FOXO3a plasmid. 

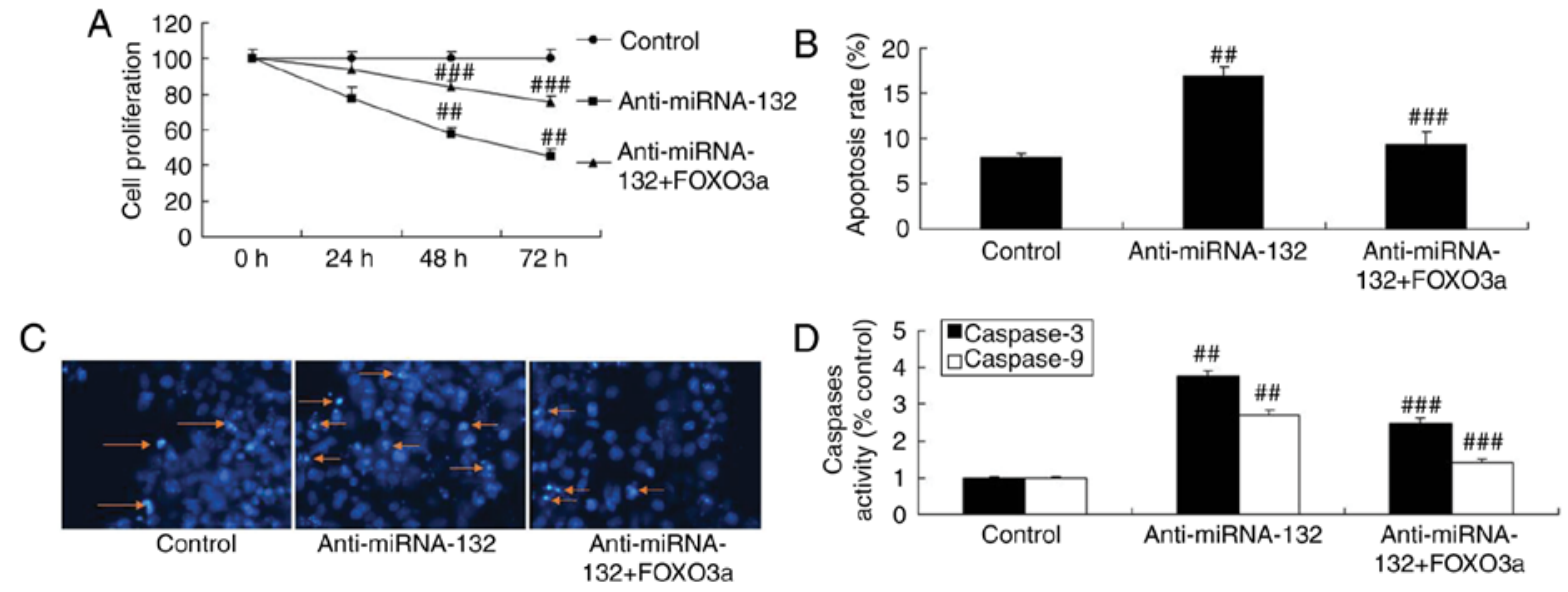

Figure 10. Promotion of FOXO3a inhibits the effects of anti-miRNA-132 on neuronal apoptosis through the AKT/FOXO3a pathway in a sevoflurane-induced in vitro model. (A) Cell proliferation and (B) cell apoptosis determined using flow and by (C) DAPI staining (magnification, x10). (D) Caspase-3/9 activity. Values are expressed as the mean \pm standard deviation $(n=3)$. ${ }^{\# \#} \mathrm{P}<0.01$, compared with the control group; ${ }^{\# \# /} \mathrm{P}<0.01$, compared with the anti-miRNA-132 group. FOXO3a, forkhead box O31; LDH, lactate dehydrogenase; miRNA, microRNA; control, negative control; anti-miRNA-132, downregulated expression of miRNA-132; anti-miRNA-132+FOXO3a, anti-miRNA-132+FOXO3a plasmid. Arrows indicate apoptotic nuclei.

apoptosis (3). In addition, they are accompanied with definite long-term cognitive and memory impairment (4). Neuronal apoptosis is considered a potential mechanism of cell injury. The activated caspase- 3 can damage the corresponding cytoplasm and nuclear substrate during early apoptosis, thus inducing cell apoptosis. The results of the present study showed that the expression of miRNA-132 was downregulated in sevoflurane-induced rats, compared with that in the normal group. The downregulated expression of miRNA-132 induced neuronal cell apoptosis in the sevoflurane-induced in vitro model.

There are numerous downstream effector molecules of PI3K. Of these, Akt is the central link in the pathway (16). The activation of Akt can act on multiple targets, including the downstream NF- $\mathrm{KB}$ family, Bcl-2 family and caspase protein family. It loses activity through phosphorylating the apoptotic protein, enhancing the transcription and expression of apoptotic genes (17). The decreased phosphorylation of Akt downregulates the expression of $\mathrm{Bcl}-2$ and upregulates the expression of Bax in the cytoplasm. This was observed in Akt gene-knockout mice and further aggravated the degree of brain damage (17). The results of the present study indicated that the downregulation of miRNA-132 induced the expression of Bax and caspase-3/9, and promoted the PI3K/AKT/FOXO3a pathway in the sevoflurane-induced in vitro model. Lian et al demonstrated that the overexpression of miR-132 enhanced cell proliferation and tumor growth in laryngeal squamous cell carcinoma through the PI3K/AKT/FOXO1 pathway (18). Zhang et al showed that miRNA-132 attenuated the neurobehavioral and neuropathological changes in mice with intracerebral hemorrhage (19). These results indicate that miRNA-132 attenuates sevoflurane-induced neuronal apoptosis, and its mechanism requires further investigation.

The PI3K/AKT signaling pathway is one of the important intracellular signal transduction pathways (7). It is mainly involved in regulating processes, including cell growth, differentiation, metabolism and apoptosis (20). It can also affect the activity of numerous downstream target genes and target proteins. Therefore, it can enhance cell survival (6), inhibit cell apoptosis and exert brain protective effects. Its role in mental health disease has attracted extensive attention (6). Notably, the present study found that the PI3K inhibitor increased the effects of anti-miRNA-132 on neuronal apoptosis through the PI3K/AKT/FOXO3a pathway in the sevoflurane-induced in vitro model. Qi and Zhang also showed that miRNA-132 regulates fluid shear stress-induced differentiation in periodontal ligament cells through the PI3K/AKT/mammalian target of rapamycin signaling pathway (21).

FoxO3a is an important downstream target of the PI3K/Akt signaling pathway. It is important in the anti-apoptotic mechanism of insulin-like growth factor-1 (22). The dephosphorylation of FoxO3a can affect the Bcl-2 family genes, pre-apoptotic gene Bcl-2-interacting mediator of cell death and growth arrest. In addition, it can affect the DNA damage-inducible gene Gadd $45 \alpha$ and non-specific cyclin-dependent kinase inhibitor P27kip1 (22). Therefore, it can induce cell apoptosis. Activation of the PI3K/Akt pathway can phosphorylate FoxO3a, which loses its transcription activity (20). Finally, it can reduce neuronal apoptosis. FoxO3a is a transcription factor, which is located in the cell nucleus. It can bind with the response elements on DNA and activate target genes (23). It is also involved in regulating cell proliferation, differentiation, metabolism and apoptosis (23). It has been suggested that FoxO3a can upregulate the expression of cell death-related genes, including P53, which indicates that FoxO3a is regulated by the PI3K/Akt pathway (24). The present study showed that the promotion of FOXO3a inhibited the effects of anti-miRNA-132 on neuronal apoptosis through the AKT/FOXO3a pathway in a sevoflurane-induced in vitro model (25). miRNA-132 may have a neuroprotective effect against sevoflurane-induced neuronal apoptosis through the PI3K/AKT/FOXO3a pathway.

In conclusion, the present study provided evidence that the expression of miRNA-132 was downregulated in sevoflurane-induced rats, compared with that in normal rats. The inhibition of miRNA-132 caused sevoflurane-induced neuronal apoptosis via the PI3K/AKT/FOXO3a pathway. The miRNA-132/PI3K/AKT/FOXO3a signaling pathway responsible for sevoflurane-induced neuronal apoptosis requires elucidation in vivo in future experiments. 


\section{Acknowledgements}

Not applicable.

\section{Funding}

This study was supported by the Major Project of Science and Technology of Shandong Province (grant no. 2016GSF201070).

\section{Availability of data and materials}

The analyzed data sets generated during the study are available from the corresponding author on reasonable request.

\section{Authors' contributions}

BY designed the experiments. PD, XD, JZ, DL and LL performed the experiments. BY and PD analyzed the data. BY wrote the manuscript. All authors read and approved the final manuscript.

\section{Ethics approval and consent to participate}

The study protocol was approved by the Animal Use and Care Committee for Research and Education of Shandong University (Shandong, China).

\section{Patient consent for publication}

Not applicable.

\section{Competing interests}

The authors declare that they have no competing interests.

\section{References}

1. Tian Y, Guo S, Wu X, Ma L and Zhao X: Minocycline alleviates sevoflurane-induced cognitive impairment in aged rats. Cell Mol Neurobiol 35: 585-594, 2015.

2. Yang ZJ, Wang YW, Li CL, Ma LQ and Zhao X: Pre-treatment with a Xingnaojing preparation ameliorates sevoflurane-induced neuroapoptosis in the infant rat striatum. Mol Med Rep 11: $1615-1622,2015$

3. Yufune S, Satoh Y, Akai R, Yoshinaga Y, Kobayashi Y, Endo S and Kazama T: Suppression of ERK phosphorylation through oxidative stress is involved in the mechanism underlying sevoflurane-induced toxicity in the developing brain. Sci Rep 6: 21859, 2016.

4. Tian Y, Wu X, Guo S, Ma L, Huang W and Zhao X: Minocycline attenuates sevoflurane-induced cell injury via activation of Nrf2. Int J Mol Med 39: 869-878, 2017.

5. Xiang J, Pan J, Chen F, Zheng L, Chen Y, Zhang S and Feng W: L-3-n-butylphthalide improves cognitive impairment of APP/PS1 mice by BDNF/TrkB/PI3K/AKT pathway. Int J Clin Exp Med 7: 1706-1713, 2014.

6. Zhu G, Wang X, Wu S and Li Q: Involvement of activation of $\mathrm{PI} 3 \mathrm{~K} / \mathrm{Akt}$ pathway in the protective effects of puerarin against $\mathrm{MPP}^{+}$-induced human neuroblastoma SH-SY5Y cell death. Neurochem Int 60: 400-408, 2012.

7. Hossini AM, Quast AS, Plötz M, Grauel K, Exner T, Küchler J Stachelscheid H, Eberle J, Rabien A, Makrantonaki E and Zouboulis CC: PI3K/AKT signaling pathway is essential for survival of induced pluripotent stem cells. PLoS One 11: e0154770, 2016

8. Liao R, Yan F, Zeng Z, Farhan M, Little P, Quirion R, Srivastava LK and Zheng W: Amiodarone-induced retinal neuronal cell apoptosis attenuated by IGF-1 via counter regulation of the PI3k/Akt/FoxO3a pathway. Mol Neurobiol 54 6931-6943, 2017.
9. Chmielarz P, Konovalova J, Najam SS, Alter H, Piepponen TP, Erfle H, Sonntag KC, Schütz G, Vinnikov IA and Domanskyi A: Dicer and microRNAs protect adult dopamine neurons. Cell Death Dis 8: e2813, 2017.

10. Yoo HI, Kim BK and Yoon SK: MicroRNA-330-5p negatively regulates ITGA5 expression in human colorectal cancer. Oncol Rep 36: 3023-3029, 2016.

11. Wang H, Li XT, Wu C, Wu ZW, Li YY, Yang TQ, Chen GL, Xie XS, Huang YL, Du ZW and Zhou YX: miR-132 can inhibit glioma cells invasion and migration by target MMP16 in vitro. Onco Targets Ther 8: 3211-3218, 2015.

12. Leinders M, Üçeyler N, Pritchard RA, Sommer C and Sorkin LS: Increased miR-132-3p expression is associated with chronic neuropathic pain. Exp Neurol 283: 276-286, 2016.

13. Yao C, Shi X, Zhang Z, Zhou S, Qian T, Wang Y, Ding F, Gu X and $\mathrm{Yu}$ B: Hypoxia-induced upregulation of miR-132 promotes schwann cell migration after sciatic nerve injury by targeting PRKAG3. Mol Neurobiol 53: 5129-5139, 2016.

14. Livak KJ and Schmittgen TD: Analysis of relative gene expression data using real-time quantitative PCR and the 2(-Delta Delta C(T)) method. Methods 25: 402-408, 2001.

15. Zhou ZB, Yang XY, Tang Y, Zhou X, Zhou LH and Feng X: Subclinical concentrations of sevoflurane reduce oxidative stress but do not prevent hippocampal apoptosis. Mol Med Rep 14: 721-727, 2016.

16. Guo XQ, Cao YL, Hao F, Yan ZR, Wang ML and Liu XW: Tangeretin alters neuronal apoptosis and ameliorates the severity of seizures in experimental epilepsy-induced rats by modulating apoptotic protein expressions, regulating matrix metalloproteinases, and activating the PI3K/Akt cell survival pathway. Adv Med Sci 62: 246-253, 2017.

17. Luo T, Liu G, Ma H, Lu B, Xu H, Wang Y, Wu J, Ge P and Liang J: Inhibition of autophagy via activation of PI3K/Akt pathway contributes to the protection of ginsenoside $\mathrm{Rb} 1$ against neuronal death caused by ischemic insults. Int J Mol Sci 15: 15426-15442, 2014.

18. Lian R, Lu B, Jiao L, Li S, Wang H, Miao W and Yu W: MiR-132 plays an oncogenic role in laryngeal squamous cell carcinoma by targeting FOXO1 and activating the PI3K/AKT pathway. Eur J Pharmacol 792: 1-6, 2016.

19. Zhang Y, Han B, He Y, Li D, Ma X, Liu Q and Hao J: MicroRNA-132 attenuates neurobehavioral and neuropathological changes associated with intracerebral hemorrhage in mice. Neurochem Int 107: 182-190, 2017.

20. Meng Y, Wang W, Kang J, Wang X and Sun L: Role of the PI3K/AKT signalling pathway in apoptotic cell death in the cerebral cortex of streptozotocin-induced diabetic rats. Exp Ther Med 13: 2417-2422, 2017.

21. Qi L and Zhang Y: The microRNA 132 regulates fluid shear stress-induced differentiation in periodontal ligament cells through mTOR signaling pathway. Cell Physiol Biochem 33: 433-445, 2014

22. Zeng Z, Wang X, Bhardwaj SK, Zhou X, Little PJ, Quirion R, Srivastava LK and Zheng W: The atypical antipsychotic agent, clozapine, protects against corticosterone-induced death of PC12 cells by regulating the $\mathrm{Akt} / \mathrm{FoxO} 3 \mathrm{a}$ signaling pathway. Mol Neurobiol 54: 3395-3406, 2017.

23. Liu MH, Yuan C, He J, Tan TP, Wu SJ, Fu HY, Liu J, Yu S, Chen YD, Le QF, et al: Resveratrol protects PC12 cells from high glucose-induced neurotoxicity via $\mathrm{PI} 3 \mathrm{~K} / \mathrm{Akt} / \mathrm{FoxO} 3$ a pathway. Cell Mol Neurobiol 35: 513-522, 2015.

24. Zhu W, Bijur GN, Styles NA and Li X: Regulation of FOXO3a by brain-derived neurotrophic factor in differentiated human SH-SY5Y neuroblastoma cells. Brain Res Mol Brain Res 126: 45-56, 2004.

25. Kim HY, Kwon HY, Ha Thi HT, Lee HJ, Kim GI, Hahm KB and Hong S: MicroRNA-132 and microRNA-223 control positive feedback circuit by regulating FOXO3a in inflammatory bowel disease. J Gastroenterol Hepatol 31: 1727-1735, 2016.

This work is licensed under a Creative Commons Attribution-NonCommercial-NoDerivatives 4.0 International (CC BY-NC-ND 4.0) License. 